\title{
Mucosa-Associated Lymphoid Tissue Lymphoma Presenting as Massive Gastrointestinal Bleeding: A Case Report
}

\author{
Kalyan Kanneganti Bhavna Balar \\ Division of Gastroenterology, Bronx-Lebanon Hospital Center, Bronx, N.Y., USA
}

\section{Key Words}

Mucosa-associated lymphoid tissue lymphoma - Malignant lymphoma of the colon . Massive gastrointestinal bleeding

\begin{abstract}
We present a 71-year-old man with mucosa-associated lymphoid tissue (MALT) lymphoma of the colon presenting with massive gastrointestinal hemorrhage. At laparotomy two areas of irregular mucosa were noted in the ascending colon and cecum. Pathology revealed MALT lymphoma. MALT lymphoma of the colon is rare and the presentation as well as outcome of our case makes him unique.
\end{abstract}

\section{Introduction}

Mucosa-associated lymphoid tissue (MALT) lymphoma arises in the lymphoid tissue and is acquired in the course of chronic antigenic stimulation or chronic infection. It accounts for $7 \%$ of all newly diagnosed lymphomas and arises from various organs throughout the human body. Helicobacter pylori has been identified as a causative factor in development of gastric MALT lymphoma. In addition, other infectious agents such as Borrelia burgdorferi in cutaneous, Chlamydia psittaci in orbital, and Campylobacter jejuni in small intestinal lymphoma also have been reported to be related to the development of MALT lymphoma. The gastrointestinal tract is the most frequently involved extranodal location for MALT lymphomas and the stomach is the most common site for MALT lymphoma of the gastrointestinal tract. MALT lymphoma of the colon is very rare. To the best of our knowledge there are only two cases of colonic MALT lymphoma reported from the USA. 


\section{Case Presentation}

A 71-year-old man was admitted to our hospital with rectal bleeding. He denied associated abdominal pain, nausea, vomiting or hematemesis. He denied any previous history of gastrointestinal bleeding. His medical history included cirrhosis of the liver secondary to alcohol use, hypertension, coronary artery disease, atrial fibrillation and systolic congestive heart failure. He was not taking warfarin. He had had coronary artery bypass surgery in the past.

Initial physical examination revealed pallor. He was hemodynamically stable. Nasogastric aspiration was clear and rectal examination revealed a large amount of maroon-colored blood. He was admitted to the intensive care unit. Initial hematocrit was noted to be $11 \%$. After receiving more than 6 units of packed red blood cells, his hematocrit was still about $18 \%$. Emergency endoscopy and colonoscopy were done. Prior to the procedures bowel cleansing was done with polyethylene glycol. Upper gastrointestinal endoscopy revealed two clean based cratered ulcers in the duodenal bulb. No blood was found in the upper gastrointestinal tract. Colonoscopy revealed a large amount of fresh and altered blood with clots in the entire colon. There was no lesion identified to explain the bleeding. Despite vigorous irrigation and cleansing, colonoscopy could not be completed to the cecum as the large pool of blood and clots interfered with visualization. The patient was taken to the operating room for exploratory laparotomy. He was found to have two irregular appearing areas in the ascending colon clearly different from the surrounding colon. Right hemicolectomy was performed which controlled the bleeding. Pathology of the colon revealed MALT lymphoma characterized by marginal zone cells infiltrating the lamina propria in a diffuse pattern and colonizing the reactive germinal center (fig. 1). Immunohistochemical stains showed these cells to be reactive to CD20, CD43 and BCL-2 antibodies and nonreactive to cyclin D1, CD3, CD5, CD10, and BCL-6 antibodies, consistent with MALT lymphoma (ig. 2, fig. 3). H. pylori was negative. A hematology/oncology team evaluated the patient. Staging work-up was planned once the patient would become stable. He remained in the intensive care unit where he later developed sepsis and expired two weeks after the surgery.

\section{Discussion}

Isaacson and Wright first described MALT lymphoma in 1983 [1]. The stomach is the most common site of gastrointestinal lymphoma and constitutes about $70 \%$ of all cases. Colorectal lymphoma comprises about $10-20 \%$ of gastrointestinal lymphomas and only $0.2-0.6 \%$ of large bowel malignancies [2]. MALT lymphoma of the small and large intestine is rare [3-6]. There are case series described from Japan, China, Italy, and Singapore where they had long-term follow-up of these patients. To our knowledge only two cases of colorectal MALT lymphoma have been reported from the USA. One of the patients had only rectal lesion whereas the second patient had rectal and cecal lesion [4]. In our case, the right side of the colon was involved. The presentation of our case with massive gastrointestinal hemorrhage and adverse outcome makes him unique. We believe that associated medical conditions such as cirrhosis contributed to the postoperative complications and ultimate demise of our patient.

The presentation of colonic MALT lymphoma varies from asymptomatic, occult bleeding to gross gastrointestinal hemorrhage. Mean age described in previous series was about 59.8 years. The gross appearance of MALT lymphoma can vary from gross polypoid lesion to ulcer or simple mucosal irregularity or discoloration [7]. Histologic features of MALT lymphoma include infiltration of centrocyte-like cells, which may show plasma cell differentiation, lymphoepithelial lesions and reactive lymphoid follicles. Immunochemically, MALT lymphoma is negative for CD5 and cyclin D1.

The treatment of gastric MALT lymphoma is well established [5] whereas the treatment of colonic lymphoma is less clear. In the previous reports, it has been reported that such lesions were treated with surgery, chemotherapy, radiation and chemotherapy, endoscopic resection followed by antibiotic treatment or antibiotic treatment alone. The role of $H$. pylori and its response to antibiotics makes the treatment of gastric lymphoma 
very effective [5]. Patients who do not respond to the antibiotic therapy for gastric MALT lymphoma are treated with chemotherapy. The literature has reported that extragastric MALT lymphoma also regresses with eradication of $H$. pylori, or with antibiotic therapy even in cases where H. pylori was not detected. Contrary to such results, Grunberger et al. [8] reported that $H$. pylori eradication was ineffective for the treatment of patients with extragastric MALT lymphoma even if they were noted to have $H$. pylori infection. Thus with controversial results from various studies, so far there are no clear guidelines for the therapy of MALT lymphoma of the colon. In previous reports, most of the patients when treated surgically had been cured with variable duration of disease-free period. With surgery or chemotherapy the prognosis for patients with MALT lymphoma is generally good [9], though results from multicenter prospective studies will be needed for clear guidelines to be developed. We understand that our patient had no long-term follow-up, as he did not survive the complications that developed after the surgical treatment for his MALT lymphoma. Our patient may have had disseminated MALT lymphoma of the gastrointestinal tract as he did have ulcers in the duodenum, but this was difficult to prove without biopsy. Our patient remained unstable for staging and possible treatment.

\section{Acknowledgement}

We are grateful to Dr. Masooma Niazi for providing the histopathology slides for this case.

Fig. 1. Colonic MALT lymphoma. The marginal zone cells infiltrate the lamina propria in a diffuse pattern and have colonized the reactive germinal centers.

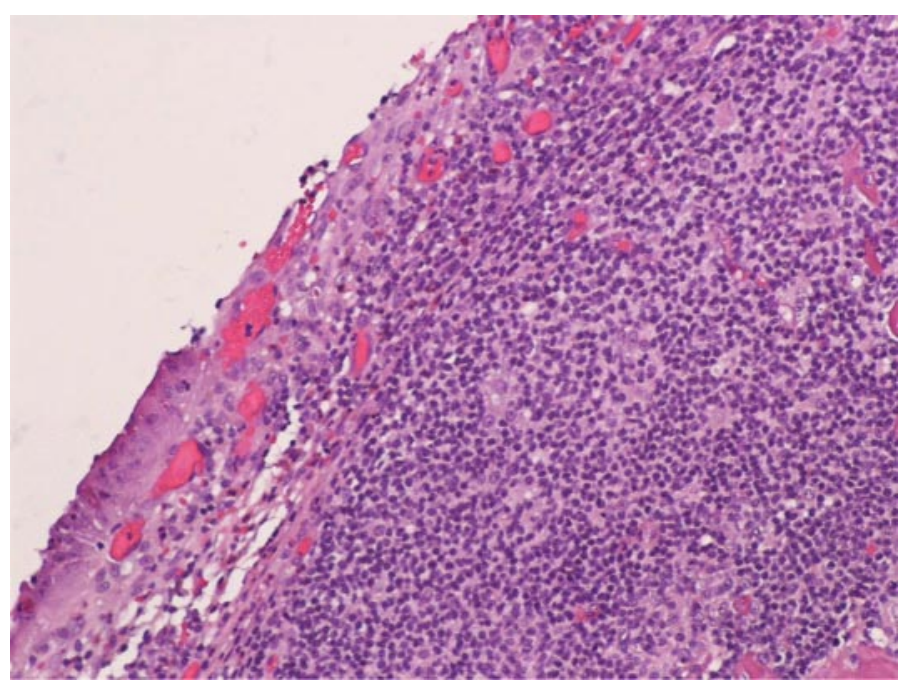




\begin{tabular}{r|l|l|l} 
Case Reports in & $\begin{array}{l}\text { Case Rep Gastroenterol 2008;2:296-300 } \\
\text { D01: 10.1159/000154814 }\end{array}$ & Published online: September 30, 2008 & $\begin{array}{l}\text { @ 2008 S. Karger AG, Basel } \\
\text { ISSN 1662-0631 } \\
\text { www.karger.com/crg }\end{array}$ \\
\hline
\end{tabular}

Fig. 2. The tumor cells show strong positivity to CD20.

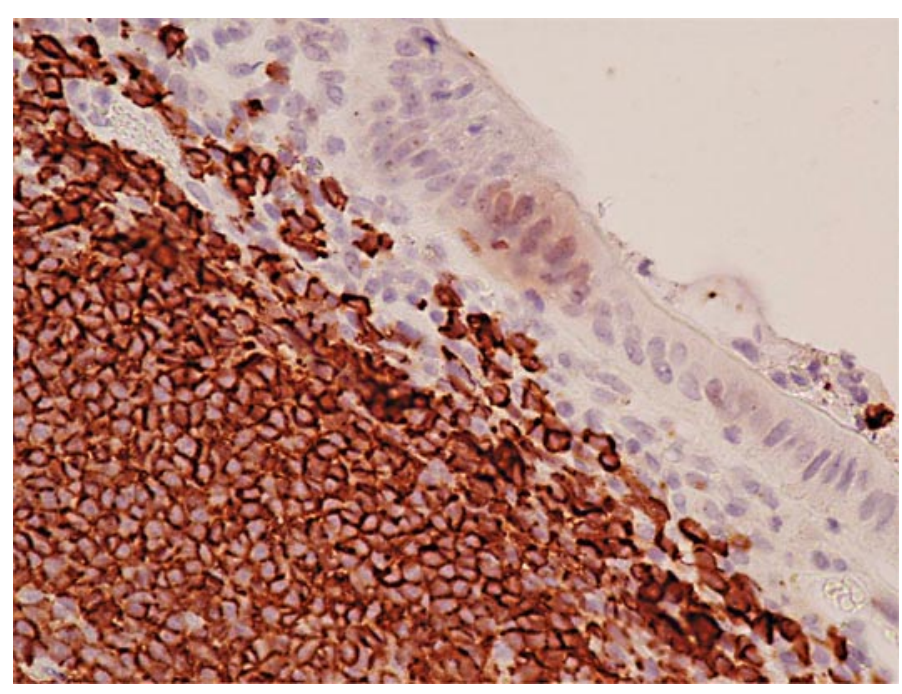

Fig. 3. Expression of BCL-2 protein in tumor cells.

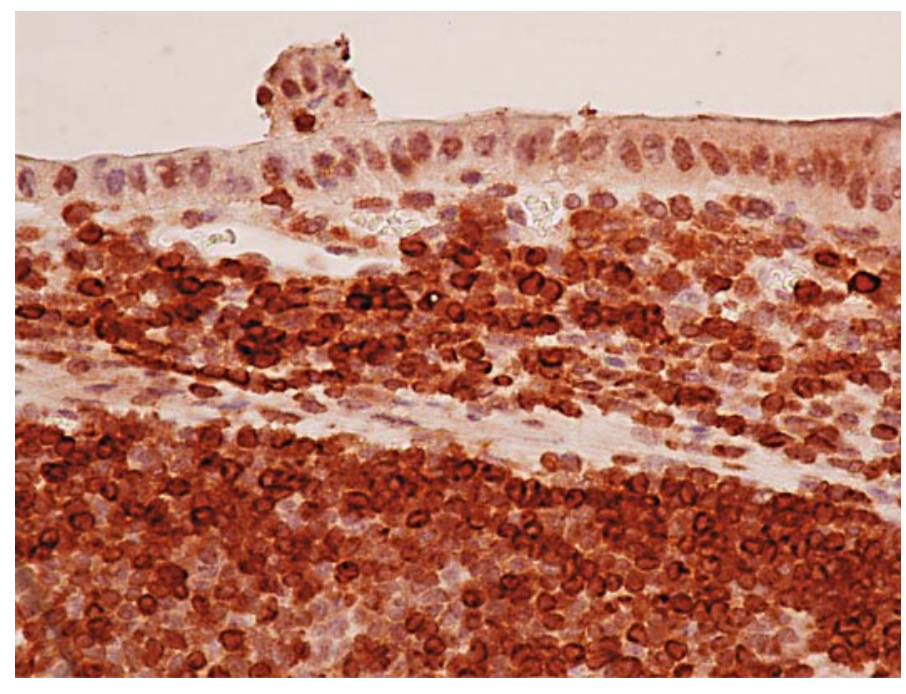




\section{References}

1 Isaacson P, Wright DH: Malignant lymphoma of mucosa-associated lymphoid tissue. A distinctive type of B-cell lymphoma. Cancer 1983;52:1410-1416.

-2 Wong MT, Eu KW: Primary colorectal lymphomas. Colorectal Dis 2006;8:586591

3 Matsuo S, Mizuta Y, Hayashi T, et al: Mucosa-associated lymphoid tissue lymphoma of the transverse colon: a case report. World J Gastroenterol 2006;12:5573-5576.

-4 Ahlawat S, Kanber Y, Charabaty-Pishvaian A, et al: Primary mucosa-associated lymphoid tissue (MALT) lymphoma occuring in the rectum: a case report and review of the literature. South Med J 2006;99:1378-1384.

5 Ahmad A, Govil Y, Frank BB: Gastric mucosa-associated lymphoid tissue lymphoma. Am J Gastroenterol 2003;98:975-986.

6 Toshima M, Aikawa K, Soga K, et al: Primary duodenal MALT lymphoma. Intern Med 1999;38:957-961.

7 Lee YG, Lee S, Han SW, Lee JS: A case of multiple mucosa-associated lymphoid tissue (MALT) lymphoma of the colon identified as simple mucosal discoloration. J Korean Med Sci 2005;20:325-328.

8 Grunberger B, Wohrer S, Streubel B, et al: Antibiotic treatment is not effective in patients infected with Helicobacter pylori suffering from extragastric MALT lymphoma. J Clin Oncol 2006;24:1370-1375.

9 Li B, Shi YK, He XH, et al: Primary non-Hodgkin lymphomas in the small and large intestine: clinicopathological characteristics and management of 40 patients. Int J Hematol 2008: Epub ahead of print. 